\title{
Identification of a miRNA signature as a diagnostic and prognostic marker in renal cell carcinoma
}

\section{Muhammad Ammar Zahid, Abdelali Agouni \\ College of Pharmacy, QU Health, Qatar University mz1912625@qu.edu.qa}

\section{Background}

Renal cell carcinoma (RCC) is one of the top ten cancers worldwide and accounts for $2-3 \%$ of adult malignancies

Based on histopathological and molecular features, more than ten different subtypes of RCC are reported, but clear cell renal cell carcinoma (ccRCC) is the most common subtype and accounts for more than $80 \%$ of RCC cases.

- If diagnosed in later stages, ccRCC is associated with high renal cancer related morbidity and poor prognosis due to limited therapeutic options.

Recently, microRNAs (miRNAs) have attracted interest of the scientific community as a biomarker due to their important role in cancer development and progression.

- Availability of big epigenomic, genomic, transcriptomic, and proteomic data in the cancer genome atlas (TCGA) coupled with the advancing science of data mining have revolutionized the identification of robust diagnostic and prognostic signatures in different types of cancers.

\section{Aim of the Study}

- The aim of this study is to utilize the miRNA sequencing data of CCRCC patients to identify a diagnostic and prognostic signature by using a combined approach of differential expression analysis, survival analysis and machine learning

- The biological significance of the identified signature will also be analyzed which will help better understand the disease process and opportunity of new therapeutic interventions.

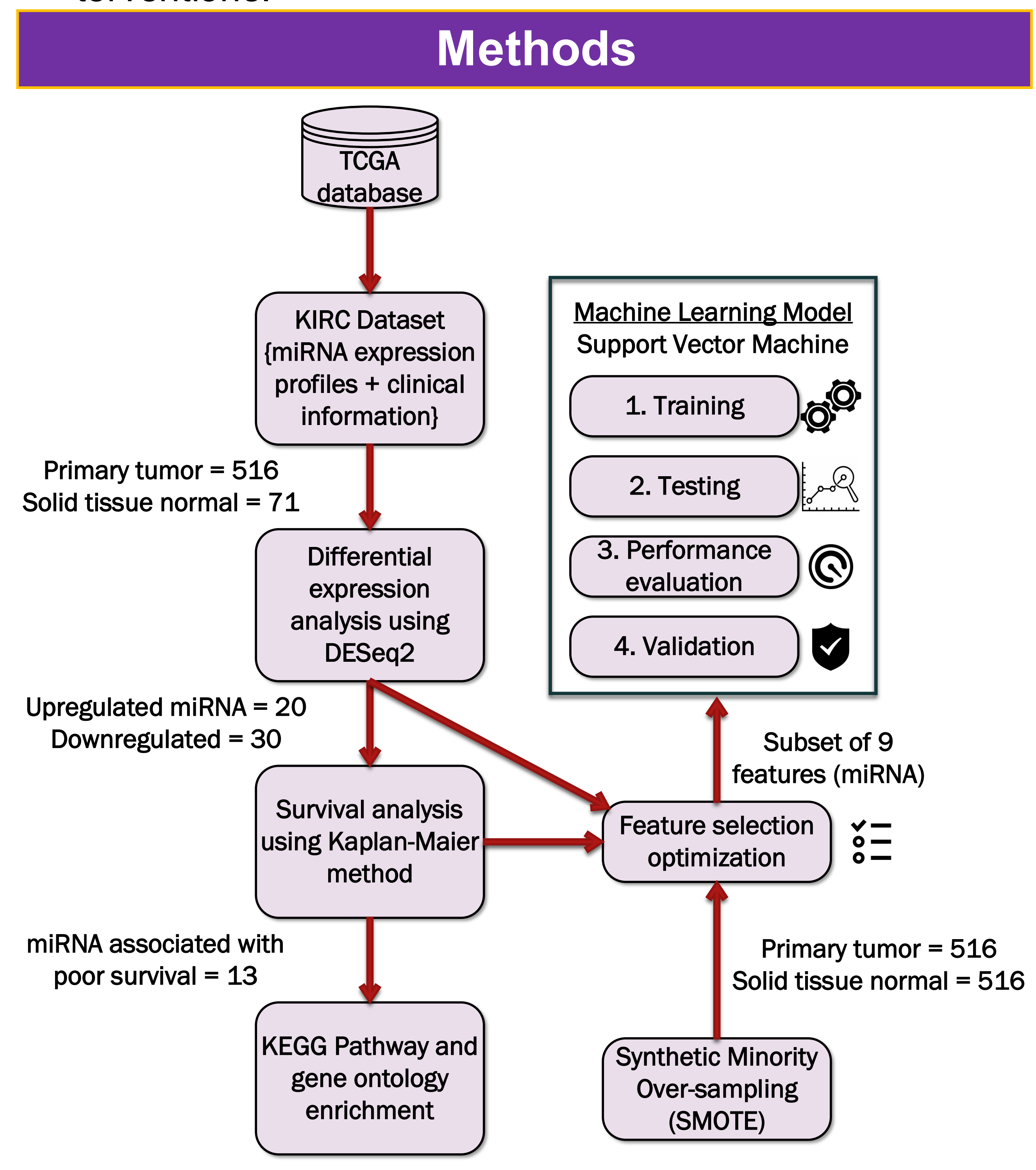

\section{Conclusions and Future Direction}

In this study we have identified a nine-miRNA signature in ccRCC patients by the combined approach of differential expression analysis, survival analysis and machine learning. Model based on this signature is capable of classifying tumor samples from solid tissue normal samples with high accuracy and precision. The validation of this classification model in a clinical cohort is the next logical step to trans-
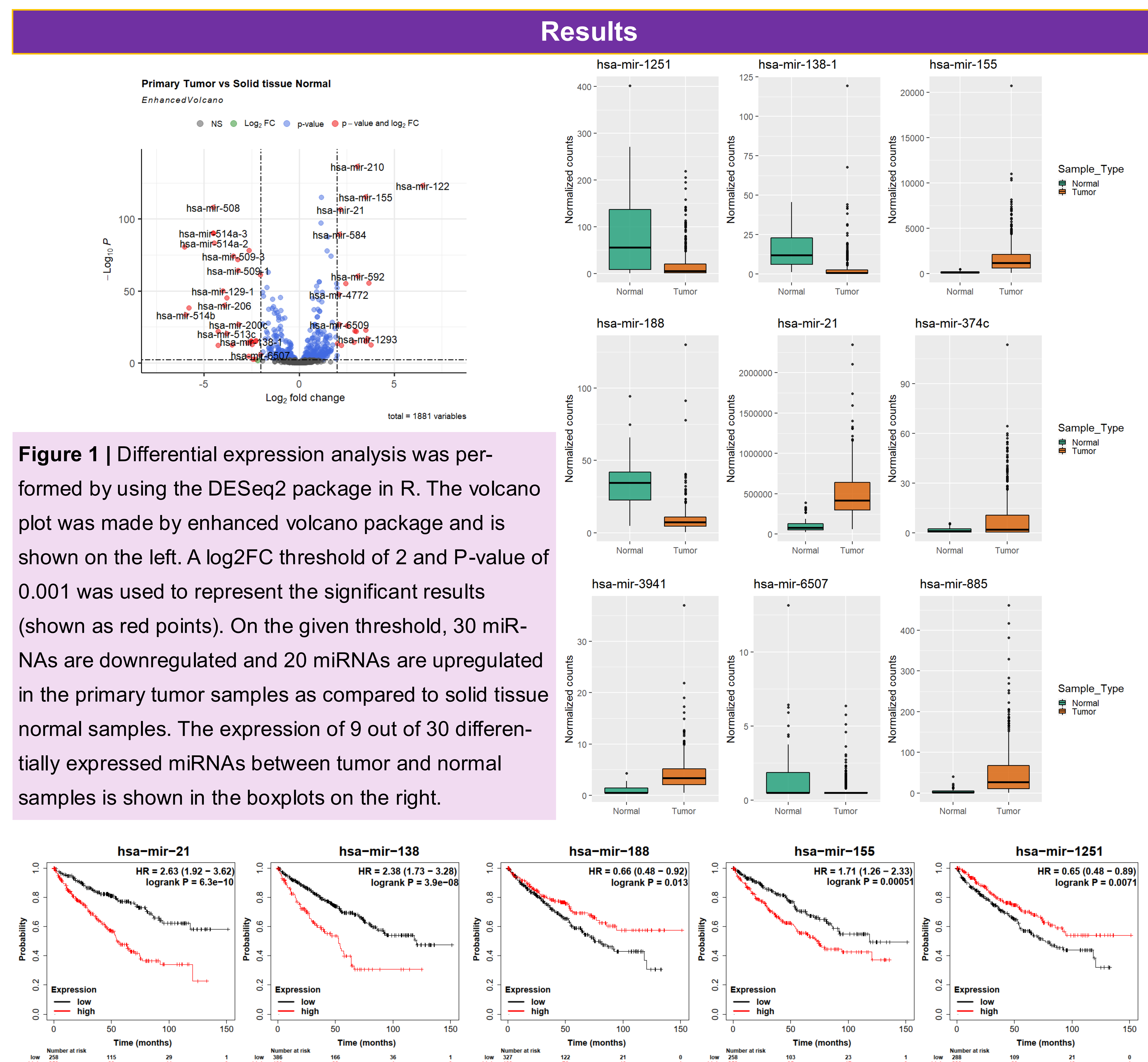

Figure 2 | The Kaplan-Maier survival analysis of the differentially expressed miRNAs was performed and the representative KM survival plots are presented here. Out of 50 differentially expressed miRNAs, higher expression of 7 and lower expression of 6 miRNAs was found to be significantly associated with poor survival $(P<0.05)$.
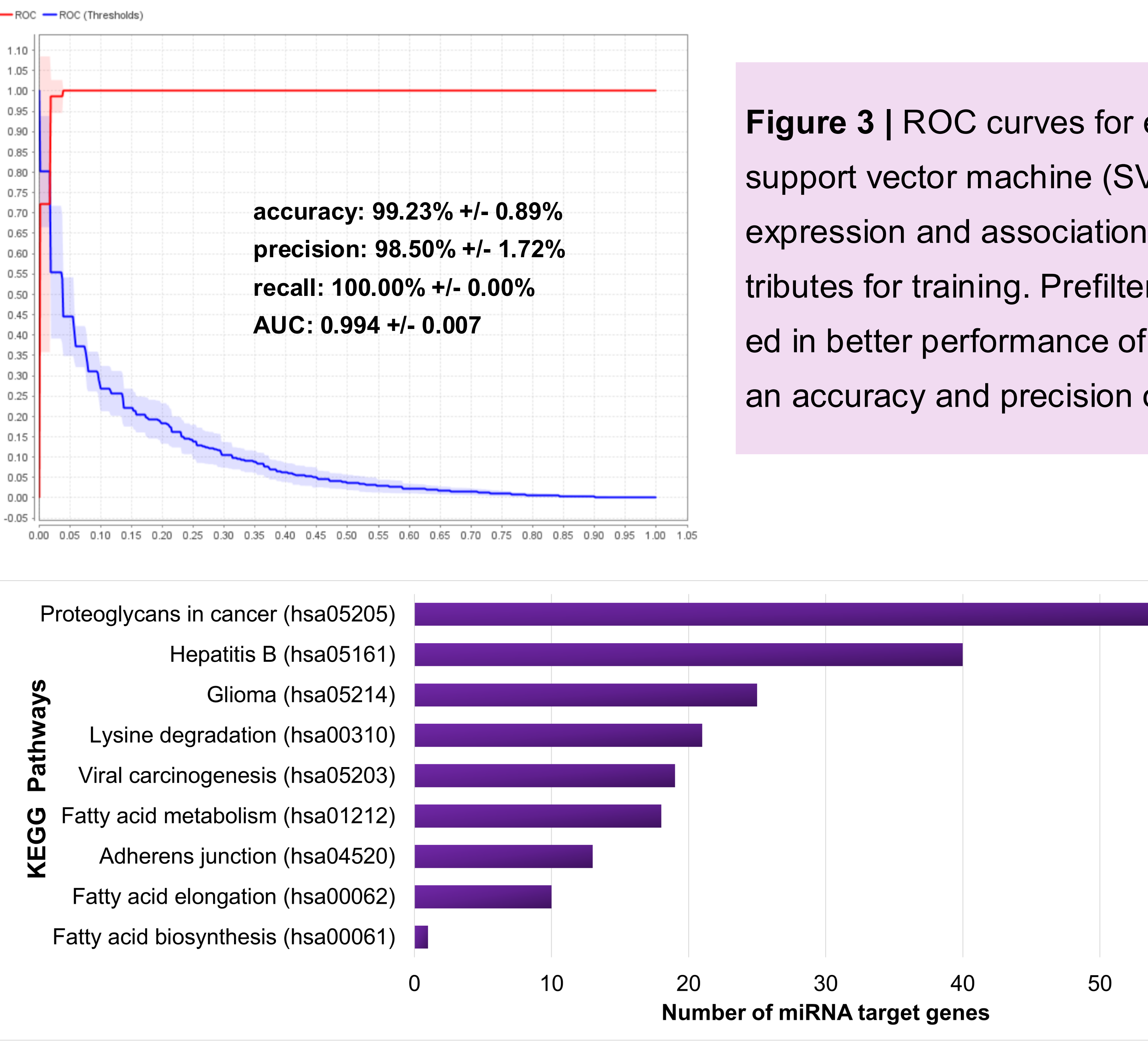

Figure 4 | The KEGG pathways enriched in targets of the miRNA associated with poor survival are shown in the vertical dimension, and the number of target genes involved in pathways are represented by vertical bars (left). Fatty acid biosynthesis is the most significantly enriched pathway $(P<1 e-325)$ and proteoglycans in cancer pathway is enriched by highest number of target genes (53). A heatmap showing enriched KEGG pathways is shown (right)
Figure 3 | ROC curves for evaluating the predictive performance of the support vector machine (SVM) model developed by using differential expression and association with poor survival as a prefilter to select attributes for training. Prefilter and attribute selection optimization resulted in better performance of the model in 10 -folds cross validation with an accuracy and precision of $99.23 \%$ and $98.50 \%$ respectively. 